\title{
REFLEXIÓN EPISTEMOLÓGICA EN ESTUDIANTES DE POST TÍTULO EN FISIOTERAPIA/KINESIOLOGÍA SEGÚN EL MODELO FUNCIÓN-DISFUNCIÓN
}

\author{
Epistemological Reflection in Post-Degree Students in Physical Therapy/Kinesiology \\ According to the Function-Disfunction Model
}

\author{
Máximo Escobar-Cabello ${ }^{1}$ \\ ORCID: 0000-0001-5780-207X \\ Hernán Maureira-Pareja ${ }^{2}$ \\ ORCID: 0000-0002-4664-0287 \\ ${ }^{12}$ Universidad Católica del Maule, Chile \\ Correspondencia: maxfescobar@gmail.com; dr.hernanmaureira@gmail.com
}

Recibido: 30/05/2020

Aceptado: 23/10/2020

Resumen: Este estudio indaga la argumentación de una cohorte de estudiantes al inicio de su post título. Tratándose de profesionales que buscan mayor validación, es atendible conocer los alcances que entrega la reflexión epistemológica en un marco institucionalizado que por tradición es desfavorable. Con el consentimiento de los estudiantes del post título Función-Disfunción Ventilatoria 2018, y a través de la construcción de un ensayo modificado que recibió el apoyo previamente de contenidos cognitivos, procedimentales y actitudinales atingentes a la temática, se realizó un análisis de contenido para identificar las categorías consideradas como relevantes para la obtención de la condición de experticia profesional. En los hallazgos, se asigna y destaca el reconocimiento explícito de las bases epistemológicas tributarias a los fundamentos que organizan consecuencias y efectos sustantivos para el post título en cuestión. Finalmente, se discuten las variables relacionadas y necesarias para demarcar la especialidad y las consecuencias de su responsabilidad social.

Palabras clave: reflexión epistemológica; post título; kinesiología; necesidades educacionales; práctica profesional.

\begin{abstract}
This study investigates the argumentation of a cohort of students at the beginning of their postdegree. In the case of professionals seeking greater validation, it is worth knowing the scope that epistemological reflection provides in an institutionalized framework that is traditionally unfavorable to them. With the consent of the students of the post-title Ventilatory Dysfunction 2018 and through the construction of a modified trial that previously received the support of cognitive, procedural and attitudinal content related to the subject, a content analysis was carried out to identify the categories considered relevant to obtain the status of professional expertise. In the findings, the explicit recognition of the epistemological bases is assigned and highlighted to the foundations that organize consequences and substantive effects for the post title in question. The related and necessary variables to demarcate the specialty and the consequences of their social responsibility are discussed.
\end{abstract}

Keywords: epistemological reflection; post title; kinesiology; educational needs; professional practice. 


\section{Introducción}

En Chile el post título en fisioterapia/kinesiología es un área de acción profesional que se origina aproximadamente hace medio siglo (Rodríguez, Moreno, Plaza y Retamal, 2017). El fisioterapeuta/kinesiólogo especialista participa en proyectos y estrategias para la salud, en los diferentes contextos clínicos que presentan disfunciones del movimiento agudas o crónicas, derivadas de las afecciones del sistema ventilatorio (Artigas, 2012). Además, las tendencias epidemiológicas nacionales han reforzado esta área de la funcionalidad y el bienestar, lo cual involucra seriamente considerar el perfeccionamiento futuro de este capital humano (Goldstein et al., 2011). No obstante, los procesos de formación que materializan estos niveles de capacitación no tratan la complejidad de los fundamentos epistemológicos involucrados y curricularmente en ellos no está el propósito que visualice un plan de formación que exceda la racionalidad técnico instrumental.

$\mathrm{Si}$ bien los antecedentes concretos al respecto dan cuenta del crecimiento de las prestaciones en atención primaria - la mayor cobertura de servicios públicos las 24 horas del día los 365 del año- esto refleja, con hechos irrefutables, el grado sostenido de una efectiva actividad profesional de alta complejidad que determina un escenario de vanguardia asistencial (MuñozCofré et al., 2017).

Sin embargo, si los impactos de semejantes acciones no han sido consolidados académicamente por las universidades, llama al menos la atención que la educación superior no esté considerando el aporte de una masa crítica producto de estos niveles de progreso profesional. De ahí, suponer que la formación continua solo representa una alternativa para optimizar la empleabilidad, capturando clientes disponibles en el mercado limitándolos a la reproducción de dinámicas protocolares, reduce la esencia de una institucionalidad que por definición es inherente al desarrollo y la evolución de las competencias de una actividad profesional relevante para la comunidad subsidiaria.

En este marco referencial, es urgente implementar alternativas que permitan actualizar actuaciones que discriminen entre los beneficios de la innovación y la dotación de herramientas viables para la toma de decisiones que aseguren mayores niveles de autonomía disciplinar (Medina-González, Muñoz-Cofré, Tapia-Gallardo y Escobar-Cabello, 2014). Desde el punto de 
vista académico, en la tradición ha permanecido esta insuficiencia en los programas y en la conceptualización de los post títulos no médicos, puesto que sus logros solo se registran como un reconocimiento estimable de la productividad asistencial y no como un producto de un avance en la formación universitaria.

Según el proyecto comunitario europeo HERMES, los beneficios de establecer entrenamiento de especialidad en fisioterapia/kinesiología respiratoria son significativos (Pitta et al., 2014). Así como, en un contexto local que aglutina entidades gremiales como el Departamento Nacional de Kinesiólogos Especialistas (DENAKE), en conjunto con representantes del Colegio de Kinesiólogos de Chile (CONAKI, 2018), académicos y las sociedades científicas, se ha impulsado el reconocimiento formal a la trayectoria empírica, configurando la base para la especialidad profesional (Pecarevic-Muñoz, 2017).

Sin embargo, lo preocupante es que en ambas ofertas de capacitación se han limitado las estrategias, tanto para la discusión epistemológica como para la responsabilidad social y el soporte estatal, lo que implica restringir el respaldo político, económico e integral que va asociado a la formación de este capital humano avanzado (Chiappa y Muñoz, 2015).

En el debate circunscrito de hoy se comprende que es necesario generar programas de post título y especialidad formal que aborden los problemas epistemológicos del movimiento humano (American Physical Therapy Association, 2015). En complemento, la Confederación Mundial para Terapia Física apoya el liderazgo y la promoción de las organizaciones miembros para influir en las políticas nacionales a través de la práctica de la especialización como resultado de un conocimiento profundo, habilidades y actitudes adquiridas por un fisioterapeuta/kinesiólogo calificado en un área específica, en poblaciones de interés para el bienestar y la calidad de vida pero, sobre todo, con el sentido de una formación, operativa en los distintos contextos y con una definida pertinencia disciplinar (World Physiotherapy, 2020). Para ello se hace ineludible construir desde un modelo que oriente las actividades formativas (Maureira, 2017), no solo para darle consistencia interna, sino para justificar el desarrollo del objeto de estudio.

No obstante, estas condiciones crean un escenario caracterizado por un incremento en la demanda de educación continua formal, a pesar de que las universidades aún reflejan un bajo interés en desarrollar procesos formativos de especialidad y post título para este nivel profesional. Semejante presión para la formación exige una profunda revisión de los aspectos que son la esencia de una profesión, más que pretender dar respuestas de oportunidad para la ocupación laboral 
(Emol, 2017). Una vez más los centros universitarios en este ámbito están llamados a sostener con equilibrio estas decisiones dado que tienen un profundo efecto sobre las disciplinas y los subsidiarios de tales decisiones.

En síntesis, los programas de post título que especializan fisioterapeutas/kinesiólogos formalmente en Chile son limitados en número, a lo que se agrega la ausencia curricular de bases epistemológicas en la formación de estos y otros profesionales que se consideran diferentes respecto de lo tradicional (Gramigna, 2005).

Considerando que esta configuración se proyecta en la trayectoria del ejercicio profesional actual y futuro, se hace atendible explorar esta problemática para conocer la percepción que porta un grupo de estudiantes de especialidad, al inicio de sus procesos formativos. En este contexto de análisis, el estudio pretende aportar en la línea investigativa de la educación superior de capital humano avanzado, específicamente en el conocimiento que declaran los estudiantes de especialidad respecto de los alcances de la reflexión epistemológica en la formación técnicocientífica que les compete.

\section{Método}

El estudio utilizó una metodología mixta que se enfocó en comprender los acontecimientos desde la perspectiva de los participantes (Gibbs, 2012). En este caso, se trabajó con una cohorte de estudiantes del programa de especialidad correspondientes a la versión 2018. Previa explicación y consentimiento informado, se les solicitó que construyeran argumentativamente un ensayo con las razones por las cuales creían que debían reflexionar sobre los fundamentos de su formación.

Este estudio formó parte de un trabajo más amplio conducente al desarrollo de una tesis doctoral y utilizó el análisis de contenido cuantitativo y cualitativo a partir de los ensayos que desarrollaron estudiantes de especialidad con posterioridad a la discusión de temáticas atingentes a la reflexión epistemológica (Delgado-Domínguez y Gutiérrez, 1999). Con esos productos se trabajó con un método en que se construye inductivamente un modelo conceptual (Strauss y Corbin, 2012), sobre lo que los estudiantes describen en sus respectivos manuscritos (Figura 1). 


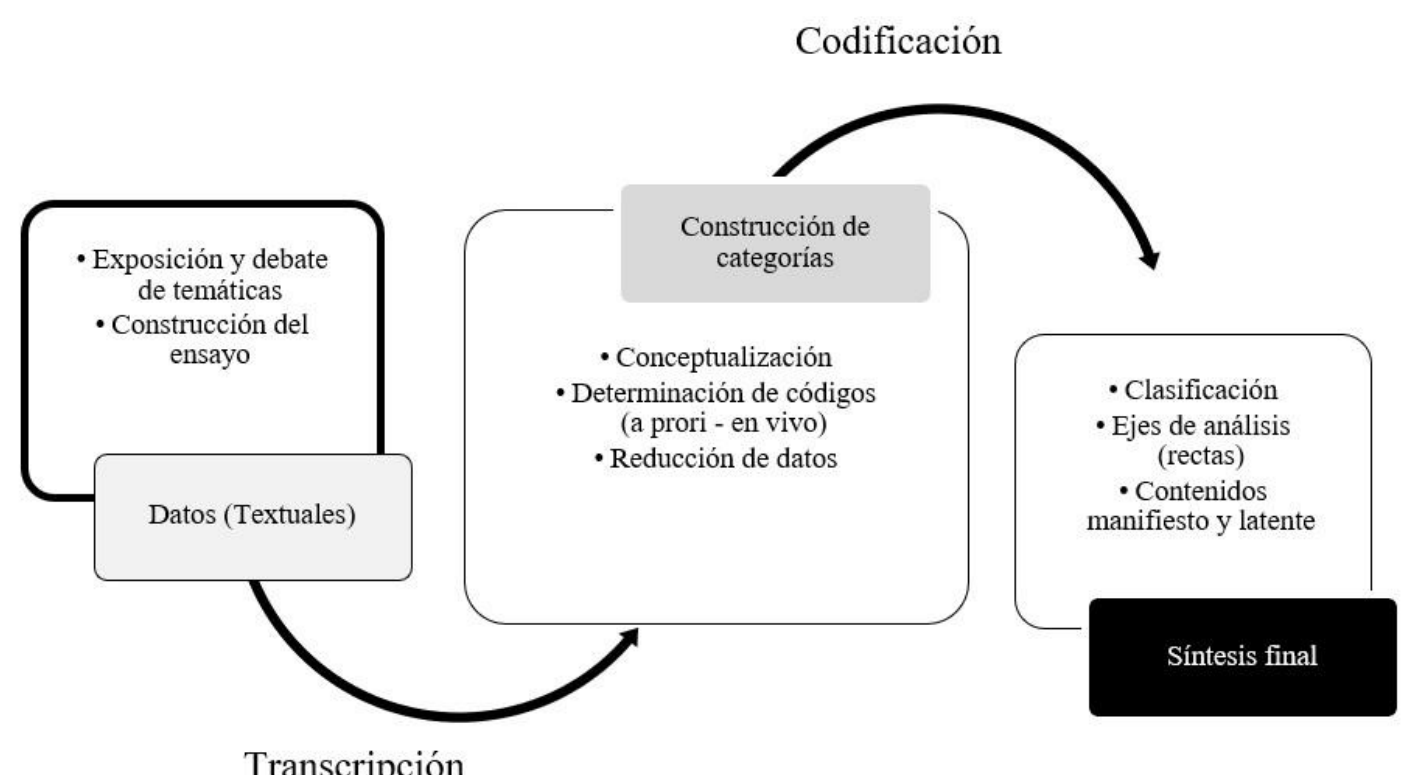

Figura 1. Desarrollo del estudio. Fuente: Elaboración propia.

\section{Participantes}

Fueron convocados a participar ocho estudiantes de especialidad pertenecientes al programa de Función y Disfunción Ventilatoria de la Universidad Católica del Maule en su tercera versión nacional, invitados con el resguardo de confidencialidad normados según acta del comité de ética científica $\left(\mathrm{N}^{\circ} 147 / 2018\right)$.

En cuanto a las características demográficas, se trata de kinesiólogos formados como licenciados provenientes de contextos clínicos que ejercen la actividad profesional en promedio 2,75 $\pm 2,87$ años. Tres de ellos trabajan de planta en hospitales públicos del país (Concepción y Purén), uno es académico de una escuela universitaria acreditada (Valdivia) y cuatro tienen trabajos en programas ministeriales de enfermedades con exacerbación estacional en los centros de salud familiar (Santiago y Talca). Siete son hombres y una es mujer; sus edades promedio son $28,2 \pm 5,2$ años; cinco son solteros y tres están casados. 
Todos presentan experiencia laboral en el campo de la atención de pacientes con disfunción ventilatoria y fueron seleccionados a partir de las postulaciones que tuvieron por requisito: a) una entrevista que dio cuenta de las razones por las cuales postulaba, b) un examen de conocimientos específicos a partir de un artículo indexado a Scielo o Scopus y c) dos cartas selladas de recomendación del candidato a especializarse.

\section{Técnicas de recogida de información}

Los datos se recolectaron a través de los manuscritos construidos por los estudiantes del programa de especialización frente a la indicación de fundamentar epistemológicamente la necesidad de especializar kinesiólogos en función y disfunción ventilatoria. Los ámbitos de interacción se desarrollaron previamente con entrega de documentos, búsqueda de información y charlas de expertos en las temáticas atingentes: Epistemología, Kinesiología, Especialización, Modelo Función-Disfunción del Movimiento Humano (MFDMH) y Operacionalización del MFDMH.

Para la estructura del producto se exigió un ensayo de 1500 palabras (letra Times New Roman, espacio simple, con un mínimo de cinco bibliografías pertinentes y referenciadas según norma APA 6). El trabajo se desarrolló con la condicionante de la orientación estandarizada del producto (rúbrica).

Para la estructura, se sugirió utilizar una modalidad recursiva escrita que planteara una tesis conocida para contra-argumentarla a partir de una antítesis personal referida a la relevancia epistemológica de la formación de post título. Definiendo esos elementos semánticos, se les solicitó preparar la resolución de la controversia con la respectiva síntesis conclusiva de un párrafo final.

Cada participante trabajó en forma individual según las indicaciones, pudiendo consultar tanto a los profesores como en la búsqueda de información que estimase apropiada. Posteriormente hicieron entrega a través del correo electrónico de sus productos con una data conocida y común para todos. Un estudiante se abstuvo de entregar el material con justificación médica. 


\section{Método de análisis de información}

Con los productos entregados se utilizó el método de análisis de contenido (AC), aproximando un preanálisis para la definición de las unidades y las reglas que permitirían la elaboración de códigos y la posterior definición de las categorías que dieran la síntesis final (Flick, 2007). Los códigos vinieron a sintetizar el conjunto de datos correspondientes a un símbolo o concepto que permitió establecer los alcances de una idea atingente a los criterios aplicados. Se obtuvieron así códigos descriptivos identificadores y códigos explicativos con mayor capacidad de inferencia.

Para la posterior elaboración de categorías que representaron la clasificación definitiva agrupando y vinculando la información, a fin de establecer un contenido manifiesto que fuera la base para la interpretación de las relaciones teóricas (Canales, 2014), se recurrió a la codificación alrededor del eje de una categoría enlazando sus propiedades y dimensiones por cada acción profesional, lo cual se conoce como rectas. De esta manera se identificaron las relaciones entre las dimensiones de las propiedades y las categorías. Estas condensaciones de códigos en principio descriptivos aseguraron que las bases de la codificación resguardaran la validez y la confiabilidad del procedimiento. Complementariamente los análisis fueron reforzados por el software Atlas.ti ${ }^{\circledR}$ versión 7 y triangulados entre los autores del estudio.

\section{Resultados}

El sistema categorial, obtenido con las concepciones establecidas por los estudiantes en sus respectivos ensayos, se construyó a partir de 29 códigos descriptores que identificaron las características distintivas. Estos, en sus propiedades y funciones, permitieron elaborar subcategorías o temáticas de mayor grado inferencial que, alrededor de sus ideas mayormente repetidas, permitieron la generación de categorías (Tabla 1). 
Tabla 1.

Acciones vinculantes con actuaciones profesionales, según los estudiantes de especialidad

\begin{tabular}{|c|c|c|}
\hline CATEGORÍAS (5) & SUB CATEGORÍAS (11) & CÓDIGOS DESCRIPTORES (29) \\
\hline $\begin{array}{l}\text { Epistemología } \\
\text { propia }\end{array}$ & $\begin{array}{l}\text { Normativas que determinan la } \\
\text { restricción del desarrollo del } \\
\text { conocimiento propio } \\
\text { Oportunidades que facilitan el } \\
\text { desarrollo del conocimiento propio }\end{array}$ & $\begin{array}{l}\text { Aspectos laborales } \\
\text { Aspectos sociales } \\
\text { Aspectos legales } \\
\text { Aspectos académicos } \\
\text { Aspectos biográficos } \\
\text { Aspectos simbólicos } \\
\text { Aspectos reflexivos }\end{array}$ \\
\hline $\begin{array}{l}\text { Fundamentos } \\
\text { disciplinares }\end{array}$ & Marco Procedimental Real & $\begin{array}{l}\text { Postura epistemológica } \\
\text { Capacidad de razonamiento } \\
\text { Operacionalización de modelos } \\
\text { Actuación profesional }\end{array}$ \\
\hline $\begin{array}{l}\text { Efectos del } \\
\text { pensamiento propio }\end{array}$ & Externos & $\begin{array}{l}\text { Construir modelos } \\
\text { Probar soluciones } \\
\text { Tomar decisiones } \\
\text { Anticipación } \\
\text { Independencia } \\
\text { Incertidumbre } \\
\text { Desafios gremiales, disciplinares }\end{array}$ \\
\hline $\begin{array}{l}\text { Herramientas } \\
\text { propias }\end{array}$ & Tradicionales & $\begin{array}{l}\text { Consolidación y materialización } \\
\text { Incremento de la participación } \\
\text { Mayor resolución de problemas } \\
\text { Sostenibilidad } \\
\text { Autonomía resolutiva }\end{array}$ \\
\hline \multirow{3}{*}{$\begin{array}{l}\text { Consecuencias } \\
\text { del accionar }\end{array}$} & Administrativas & $\begin{array}{l}\text { Ponderación de conductas } \\
\text { Procederes no neutros }\end{array}$ \\
\hline & Empleabilidad & $\begin{array}{l}\text { Desregulación } \\
\text { Cobertura de insuficiencias }\end{array}$ \\
\hline & Responsabilidad & $\begin{array}{l}\text { Respuesta a la sociedad } \\
\text { Repensar el rol }\end{array}$ \\
\hline
\end{tabular}

Fuente. Elaboración propia (2020) 
Los resultados posteriores derivaron del análisis relacional que emergió de cada una de las acciones inherentes a las temáticas declaradas por los estudiantes y fueron construidos a partir del ordenamiento conceptual y la codificación. De esta manera las categorías correspondientes que guiaron el proceso involucraron una selección de constructos teóricos que, a juicio de los estudiantes, se deberían tener presentes en un proceso de formación de especialistas. Una vez establecido el ordenamiento conceptual de los códigos, subcategorías y categorías, se seleccionaron aquellas con mayor poder de abstracción para proceder a la interpretación, basado en las percepciones por las cuales creían ellos que era atendible reflexionar sobre los fundamentos de su formación. Se muestra la frecuencia de los códigos totales que permitieron generar mayores niveles de interpretación (Gráfico 1).

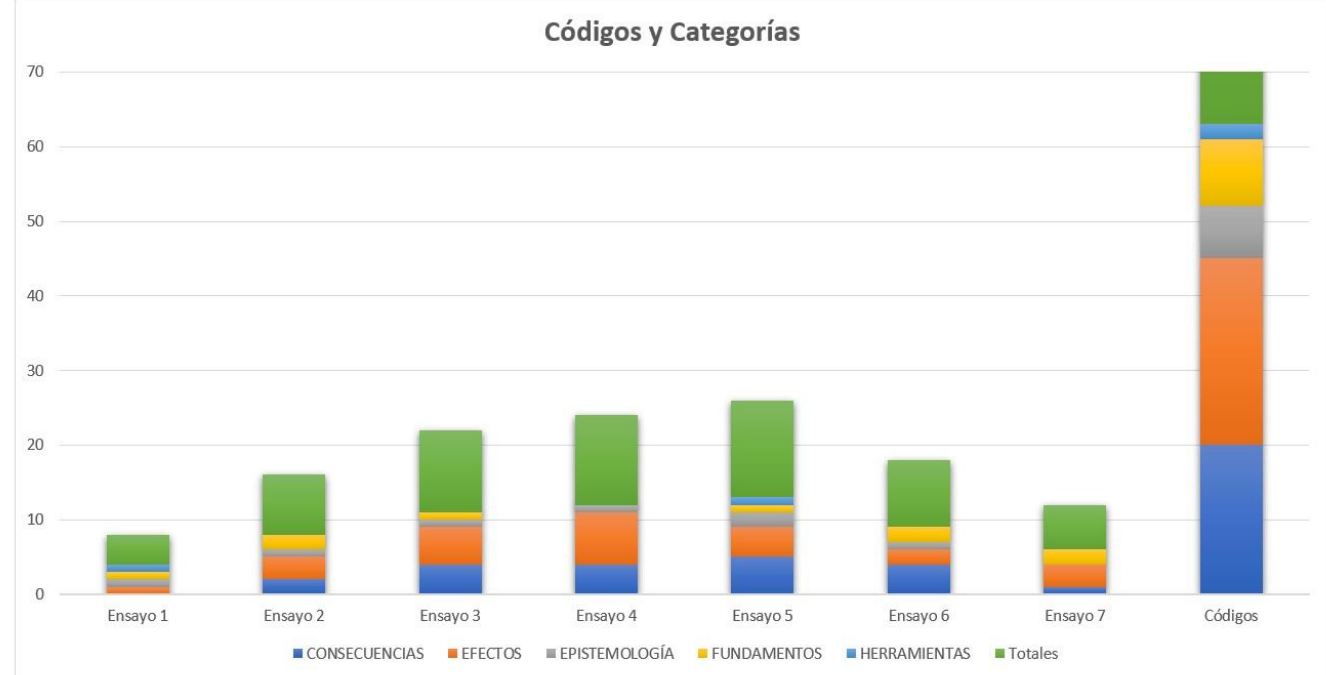

Gráfico 1. Frecuencias de las categorías obtenidas por análisis de contenido cuantitativo.

Fuente: Elaboración propia (2020)

Con el propósito de desagregar las interpretaciones de los estudiantes correspondientes a las categorías obtenidas, en cada una de ellas, además se destacan algunas de las citas textuales representativas que en sus significados establecen el predominio requerido para su justificación (Tabla 2). Estas, con la frecuencia descrita, se asocian con acciones profesionales de rutina, estandarizadas por la comunidad de los estudiantes participantes, las que terminan por dar la estructura al modelo conceptual que incorpora la totalidad de las categorías reconocidas por los estudiantes del programa de especialidad. 
Tabla 2.

Citas textuales representativas

\begin{tabular}{|c|c|}
\hline ID & Texto \\
\hline E7:2 & $\begin{array}{l}\text { "Con el fin de que se comparta una mirada epistemológica que entregue una nueva visión del abordaje, en } \\
\text { la solución de problemáticas de la sociedad actual" }\end{array}$ \\
\hline $\mathrm{E} 1: 3$ & $\begin{array}{l}\text { "El movimiento es capaz de estar presente desde su participación a nivel social [...], como en su } \\
\text { interacción microscópica celular" }\end{array}$ \\
\hline $\mathrm{E} 4: 2$ & $\begin{array}{l}\text { "La kinesiologia/ fisioterapia es en esencia una disciplina "cientifica que estudia y se especializa en el } \\
\text { movimiento y la salud humana" }\end{array}$ \\
\hline E6:6 & $\begin{array}{l}\text { "El movimiento hábil y eficiente [...], se entiende como una expresión funcional con sentido [...], lo cual } \\
\text { en su alcance condiciona la calidad de vida y la salud" }\end{array}$ \\
\hline E6:12 & $\begin{array}{l}\text { "Al gestionar las variables que favorecen el movimiento intencionado, el ciclo del flujograma de derivación } \\
\text { se rompe [...], la dependencia se termina y el objetivo inconsciente aflora al entendimiento de la salud como } \\
\text { una variable que está comulgando con función y movimiento en todo momento" }\end{array}$ \\
\hline E1:1 & $\begin{array}{l}\text { "El formar profesionales especialistas en función y disfunción ventilatoria cubre una necesidad importante } \\
\text { de entregar una atención basada en el razonamiento" }\end{array}$ \\
\hline E2:1 & $\begin{array}{l}\text { "La necesidad de formar fisioterapeutas especialistas responde a la problemática de dar soluciones } \\
\text { efectivas [...], desde un estatus de salud disfuncional o patológico" }\end{array}$ \\
\hline E7:5 & $\begin{array}{l}\text { "El conocimiento teórico nos permitirá razonar en diversas situaciones [...], será la práctica la que haga } \\
\text { al maestro y ese maestro será el encargado de decidir sobre ese paciente" }\end{array}$ \\
\hline E1:7 & $\begin{array}{l}\text { "La educación es la piedra fundamental en nuestra formación y para llegar a comprender todo de manera } \\
\text { integral debemos indagar en los diversos nichos de investigación [...] lograr responder las dudas que } \\
\text { afecten a las personas y de esta manera evidenciar que si se pueden lograr cambiar e impactar en la } \\
\text { sociedad de manera positiva" }\end{array}$ \\
\hline $\mathrm{E} 1: 8$ & "Pero para lograr esto necesariamente se debe comenzar desde el aula de pregrado" \\
\hline E6:10 & $\begin{array}{l}\text { "Un acercamiento que alivia profundamente los costos de acciones médicas (consultas repetidas por } \\
\text { exacerbación) desde el momento que contribuye a la recuperación de la calidad de vida [...] a través del } \\
\text { análisis y la asistencia del movimiento" }\end{array}$ \\
\hline $\mathrm{E} 2: 5$ & $\begin{array}{l}\text { "Es por esto que cabe la obligación educacional de realizar cambios desde cómo se están llevando a cabo } \\
\text { dichas intervenciones" }\end{array}$ \\
\hline E4:6 & $\begin{array}{l}\text { ¿Por lo mismo, la educación desde el pregrado, debiera fomentar la crítica, el cuestionamiento, la duda, } \\
\text { el por qué? }\end{array}$ \\
\hline $\mathrm{E} 4: 10$ & $\begin{array}{l}\text { "Las fallas del sistema y la incapacidad de poder entregar las herramientas epistemológicas desde la } \\
\text { educación superior..." }\end{array}$ \\
\hline E5:2 & $\begin{array}{l}\text { "Probablemente hace un par de décadas atrás y bajo un paradigma biomédico [...] la } \\
\text { kinesiologia/fisioterapia surgía a partir de la necesidad de técnicos con habilidades especificas para } \\
\text { resolver disfunciones ventilatorias" }\end{array}$ \\
\hline E5:3 & $\begin{array}{l}\text { "las necesidades de la población han ido cambiando, lo que ha dado exigencias nuevas a la profesión, } \\
\text { surgiendo asi la urgencia de ser entes generadores de conocimiento [...], con el inminente compromiso de } \\
\text { adaptarse a esos requerimientos" }\end{array}$ \\
\hline $\mathrm{E} 2: 7$ & $\begin{array}{l}\text { "La posibilidad de entregar las herramientas básicas teórico prácticas a las poblaciones que lo requieran } \\
\text { [...], acercando al profesional especialista y asimismo resolviendo los dilemas de una entrega de salud } \\
\text { insuficiente o ineficiente" }\end{array}$ \\
\hline $\mathrm{E} 7: 7$ & "resolviendo problemas en el menor tiempo posible, para empoderarse del primer contacto" \\
\hline $\mathrm{E} 7: 4$ & "La creación de nuevas especialidades es el primer gran paso para dar una atención de primer contacto" \\
\hline $\mathrm{E} 1: 2$ & $\begin{array}{l}\text { "La indagación y la puesta en marcha de nuevos conocimientos, nos entregarán mayores herramientas para } \\
\text { desenvolvernos en el mundo actual" }\end{array}$ \\
\hline E5:11 & $\begin{array}{l}\text { "Generando la gran responsabilidad de pasar de ser ejecutores a profesionales con la capacidad de } \\
\text { evaluar" }\end{array}$ \\
\hline
\end{tabular}

Fuente. Elaboración propia (2020) 


\section{Epistemología propia}

Como primera categoría en cuestión, los estudiantes de post título establecen que, dadas las circunstancias dinámicas de la profesión — cuyo desarrollo además es único en la regióndebe asumir que su historia vívida la hace poseedora de una matriz disciplinar permanentemente tensionada y a menudo restringida. Sea por los numerosos aspectos epistemológicos, sociales, laborales, legales o académicos que desde su origen han sido una constante restrictiva, convoca la necesidad de mirar retrospectiva y proyectivamente su objeto de estudio. Si bien no es la categoría de mayor densidad, en su condición básica la reconocen como un objetivo prioritario y a tener presente, dado que ya temprano en los relatos biográficos, los referentes de la profesión reconocían su relevancia trascendente. A juicio de los participantes, y como un aspecto estratégico de la formación para los estudiantes de post título, resulta crítico entender que, dada la investigación técnico-científica acumulada por la profesión aún en desarrollo, se permiten observar cada etapa como la representación de una cadena de valiosos antecedentes pertinentes a su perfeccionamiento. Es por esto que, a estas alturas de la evolución, los estudiantes de especialidad confirman que existe suficiente evidencia para profundizar aseveraciones con responsabilidad social y disciplinar (E7: 2; E1:3).

Así, interpelados quienes deciden asumir los riesgos epistemológicos, en su legítima condición de estudiosos del movimiento, tienen la libertad intelectual de establecer sus propósitos (E4:2). Se desvelan así con toda propiedad los propósitos simbólicos expresados hace algunas décadas por connotados y eméritos profesores, cuyo debate conceptual los llevó a concluir categóricamente (E6: 6). Esta declamación de alcance biopolítico estimula la modificación y el reconocimiento que determina un habitus del desempeño profesional. De esta manera, se puede constatar la magnitud de la toma de posición en contexto de un estudiante de post título, que propone abiertamente mayores niveles de integración (E6: 12).

\section{Fundamentos disciplinares}

La segunda categoría es la que responde a los argumentos que se requieren para asumir una determinada postura epistemológica y, en relación con ello, un espacio pragmático importante lo genera el marco teórico potencial que otorga la capacidad de razonamiento profesional. El razonamiento vendrá a operacionalizar el producto del pensamiento reflexivo que emerge desde la mirada epistemológica, el cual permite actuar profesionalmente en un marco procedimental de 
manera contextualizada y con un ordenamiento de las representaciones semánticas de cada una de las premisas que, heurísticamente, se asumen como reglas formales. Tales estructuras de racionalidad deben estar alineadas férreamente con las necesidades reales y a la vez optimizan la mejor gestión de soluciones (E1: 1; E2: 1).

Para ellos eso no quiere decir que solo desde la práctica se den exclusivamente los eventos que retroalimentan virtuosa y categóricamente el pensamiento. Al menos es una constatación de que regularmente la práctica nutre la reflexión en sus más íntimos simbolismos, aunque también con cierta regularidad se tienda a creer que es en la academia donde se incuban los materialismos reflexivos (E7: 5). Lo que los estudiantes afirman al declararlo es que tanto praxis como reflexión se hacen indivisibles en la interacción. Desde esta perspectiva formativa, señalan, es la toma de decisiones la herramienta que producirá el empoderamiento más cercano a la autonomía de la actuación profesional, todo ello con referencia a las intervenciones profesionales situadas y demostradas como válidas para esas problemáticas.

\section{Consecuencias del accionar}

Esta categoría involucra las derivaciones que trae aparejado el ejercicio en propiedad de un marco referencial de actuación amplio, como el que se da producto de la especialización el cual involucra la administración, la empleabilidad y la responsabilidad. La deuda de los centros de formación sobre todo en lo que respecta al capital humano es reconocido como un punto pendiente en lo que se refiere a responsabilidades sociales que se ubican por sobre la empleabilidad (E2: 5; E4: 6; E4:10). Declaran que no son argumentaciones frecuentes de encontrar en el discurso profesional, pero que, en esta etapa de la controversia, si bien las explicaciones pueden ser muy variadas y dependientes de los contextos, hoy se presenta como una urgencia no solo académica, sino administrativa (E5: 2; E5: 3).

En sus documentos no se configura ninguna desproporción al reconocer que el peso de la oferta formativa es de una desregulación absoluta. En su desequilibrio más agudo refieren que se ha permitido la proliferación de programas de post título desprovistos del más mínimo respaldo pedagógico y epistemológico. Sin embargo, en la proyección de sus escritos, lo que convoca al pensamiento reflexivo de los especialistas son otros derroteros, de otras magnitudes, que auguran perseverar a la espera de enfrentar dilemas con otras herramientas y con otros protagonistas asépticos de las leyes del mercado. Están hablando de repensar el rol en las estructuras mismas. 


\section{Efectos del pensamiento propio}

Esta categoría viene a visualizar aquellos elementos derivados indisociablemente de los beneficios obtenidos al adoptar con decisión los desafíos históricos asumidos desde la convicción epistemológica, la que se ve asistida por una nutrida base referencial sociocrítica de la evidencia que se maneja en la historia profesional.

En la perspectiva del modelo construido (Figura 2) se puede establecer que un especialista en su toma de posición epistemológica visualiza la posibilidad de recorrer un vasto y amplio espectro de contextos en los cuales se permite construir soluciones. Es más, constata que puede anticiparse de manera competente a los hechos y, desde su experticia, entregar elementos de juicio con un propósito preventivo y autorregulado. No obstante, también es consciente de que en tales escenarios existen algunas ocasiones condicionadas por la incertidumbre y la incompetencia. En esa economía de posibilidades se generan conflictos internos y también con la estructura de lo establecido externamente $y$, en su germinal contradicción, habita la posibilidad de impedir lo inevitable. Quizá sea ese el propósito central de un especialista, tomar decisiones de riesgo profesional, para lo cual se debe formar y preparar rigurosamente (E2: 7; E7: 7).

En esta consecuencia formativa, un aspecto central se define críticamente al constatar que los centros de formación cuentan con una obligación disciplinar y profesional de cobijar el avance técnico y someterlo a criterios de confiabilidad y validez procedimental para generar nuevas preguntas relevantes que generen líneas de investigación profesional. Según los estudiantes de post título, esas rutas de aprendizaje se justificarán solo en la medida en que las respuestas encontradas lleven en su conformación la independencia de un método replicable y demostrable que justifique algo más que un puesto de trabajo (E7: 4). Esta decisión, en la medida en que posibilite la obtención de estrategias viables y cargadas de responsabilidad social, activará circuitos virtuosos para la profesión y los harán ejecutantes dignos de un rol (E1: 2; E5: 11).

\section{Herramientas propias}

Un aspecto que entienden como operativo, pero no menos relevante, se configura en esta categoría, puesto que se consolidan en la materialidad de sus propuestas, las alternativas que eventualmente atacan la red de la problemática (E1:7; E1: 8). Más aún, declaran que esta materialidad continúa siendo academicista y debe configurarse en concreto porque no deja de ser controversial en su carácter tradicional hegemónico (E6: 10). 


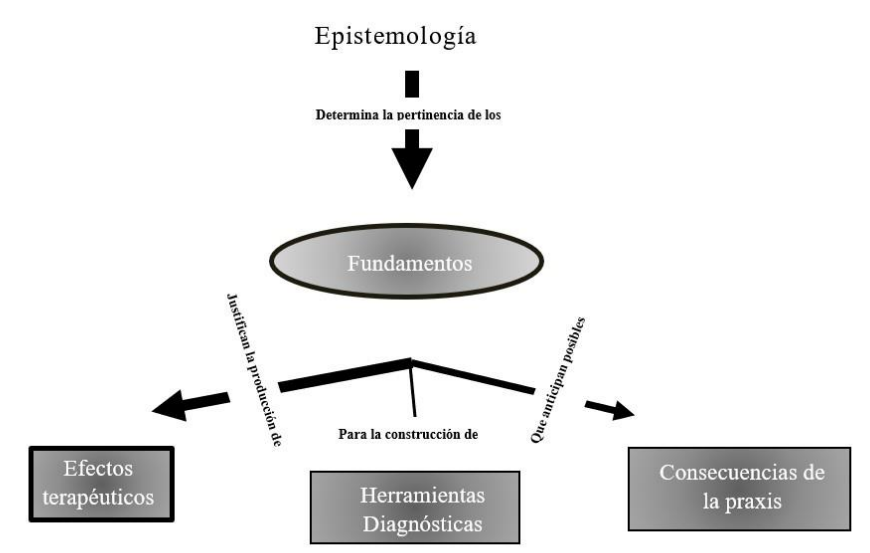

Figura 2. Ordenamiento de saberes para estructurar un programa de especialidad en kinesiología. Fuente: Elaboración propia (2020)

\section{Discusión}

El post título en la tradición refiere con cierta sistematicidad a la racionalidad técnica que se adquiere con el ejercicio experiencial de una determinada práctica. Sin embargo, la mejora automatizada de la tecnología, con el propósito de disminuir las cargas de trabajo humano, incubaron la suma de un nuevo y gran dilema, provocando una alta dependencia del manejo de información encriptada y con flujogramas protocolizados que invitan a la comodidad de reducir los procesos reflexivos de los profesionales (Vite-Pérez, 2000). Es así que un especialista se consolida como un productivo generador de acertadas respuestas efectivas, independientemente de la contingencia y la magnitud de los estereotipos.

$\mathrm{Al}$ interactuar en su formación con los elementos inherentes a su objeto de estudio - $-\mathrm{y}$ que se conceptualizaron con la categoría epistemología propia, referida en los ensayos- lograron visualizar un elemento estratégico que está presente en la trayectoria histórica de la profesión y que forma parte de los elementos simbólicos de mayor significado por sus formadores. Sin duda que el reconocimiento de la importancia de la formación para la generación de nuevo conocimiento y como espacio para el desarrollo social es de importancia académica (De Ibarrola, Sañudo, Moreno y Barrera, 2012). No obstante, en países fuertemente colonizados, es probable que en realidad vaya a ser muy lenta la profundización paradigmática en los programas de especialidad y post título, fundamentalmente porque las generaciones precedentes de kinesiólogos formadores se modelaron bajo el amparo positivista de la salud. Pero no se puede desconocer que la independencia de pensamiento es algo más que ser interpretado por otros y descansa 
indefectiblemente en el ejercicio de una praxis real (Schön, 2010). Cualquier aspiración de autorregulación profesional, sugiere desarrollar reflexión propia "en" y "sobre" la acción, masa crítica que pueda reconocer la necesidad de contar con una base epistemológica propia, una toma de posición política y, lo consecuentemente más tardío de arribar, la aceptación material y el reconocimiento formal por parte de la comunidad frente a sus demandas.

Como en todos los procesos de formación profesional, los estudiantes manifiestan la necesidad del uso clave de fundamentos disciplinares. La consecuencia operativa que itera esta categoría refuerza el que a cada acción profesional le pertenece un cúmulo de evidencias que se movilizan como recursos para respaldar la precisión de la reflexión y la pertinencia de su acción. Lo relevante es que semejantes construcciones fortalecen el juicio clínico y contribuyen al reconocimiento formal de una racionalidad operativa y la necesidad de especialización en el post título para garantizar esta capacidad (Goldstein et al., 2011). Esto se expresa en una competencia central de pensamiento profesional conocida como razonamiento. Lo contradictorio es que los programas de post título no consideran la planificación estructurada de tales competencias, al considerarlas implícitas, y son reemplazadas por aspectos técnicos como el manejo de la evidencia que disminuyen la posibilidad de generar un profesional integral y resolutivo.

Paradójicamente, la Organización Panamericana de la Salud (2019), en sus recomendaciones para la salud pública de las Américas en el siglo XXI, propuso desarrollar y fortalecer las competencias clínicas y de gestión del recurso humano profesional que participa en salud. Entre estas acciones estaba favorecer el desarrollo de especialidades clínicas, así como lograr una mayor cobertura de programas académicos relacionados con la investigación, dado que la evidencia científica correlacionaba positivamente la especialización clínica con el costo/efectividad de las prestaciones de salud. También, la formación en gestión y política pública de los profesionales de las facultades de salud son lineamientos orientados a enriquecer el capital humano del sector, de manera que impactara los indicadores sanitarios y financieros (Atria, 2014).

Sin embargo, en Chile esta acción respondió con un grado evidente de discriminación, creyendo que es una potestad estructural de ejercicio del poder establecido para conformar y mantener un marco de exclusión histórica respecto de disciplinas que no necesariamente comparten el paradigma dominante (Althusser, 1989). Es un hecho que a la fecha solo las especialidades médicas-odontológicas cuentan con planificación, financiamiento, retorno y estatus (Román y Señoret, 2008). 
De esta manera al configurar la categoría consecuencias del accionar se comienza a visualizar con mayor nitidez que las conductas administrativas en la academia simplemente no atendieron la relevancia de las propias actuaciones al interior de sus respectivas facultades. Se cristaliza el reconocimiento de que en las acciones profesionales no hay procederes inocuos o anodinos. Así es legítima la pregunta: ¿qué razón ha impedido a las unidades de kinesiología plantearse seriamente la especialización como un propósito fundamental del post título?

Cuando en la reflexión un punto a considerar para la evolución de la profesión en su quehacer se alude a la creencia de méritos consecuentes, estas respuestas aún no logran consensos mínimos, sobre todo cuando se permanece fiel al marco de referencia tradicional y, desde el cual, esa perspectiva explicativa con alta probabilidad es pertinente.

Es así como a partir de la promulgación, por parte del Ministerio de Salud, del Reglamento de Especialidades Médicas y Odontológicas, en noviembre de 2008, surgió un nuevo escenario gremial: impulsar el reconocimiento certificado de otras especialidades y de especialistas relevantes para el manejo sanitario, propendiendo con ello al mayor posicionamiento técnico y social de los profesionales denominados peyorativamente "no médicos o de colaboración médica". Estos, siendo la mayoría de la fuerza de trabajo, son invisibilizados por las estructuras formales de poder (Jara, 2019).

La presión colegiada de los diferentes gremios de terapeutas, sicólogos, farmacéuticos, tecnólogos, matronas, veterinarios, trabajadoras sociales, nutricionistas y enfermeras han extremado recursos para discutir el resguardo de saberes considerados propios, conduciendo a formular inclusive un proceso diagnóstico que evidentemente pone en el escenario un problema reproducido y arrastrado por muchos años de control (Bourdieu y Passeron, 1995) que se dilata en exceso (Ministerio de Salud, 2019). Tales acciones se enmarcan dentro de un recorrido principalmente legista que pretende un reconocimiento legal, como se ejemplifica en la pretensión de la Asociación Nacional de Acreditación de Kinesiólogos Especialistas, con Personalidad Jurídica No 157121 obtenida a partir del 26 de noviembre de 2013; aunque la táctica determina que con prioridad se debe lograr el propósito principal, modificando sustantivamente las restricciones del código sanitario que rige las prácticas desde 1937 a la fecha (Ley 725, 2019).

Entonces, según los relatos, al igual que 15 profesiones universitarias involucradas en el control sanitario de Chile, la especialidad en fisioterapia/kinesiología es un área administrativa de acción profesional pendiente. Desde ahí avanza, optimizando competencias al servicio de sus 
prácticas y afianzando los fundamentos científicos correspondientes (Rodríguez et al., 2017). Actualmente el kinesiólogo especialista participa en los diferentes contextos clínicos dirigidos a usuarios que presentan disfunciones agudas o crónicas, derivadas de las afecciones de los sistemas al servicio del movimiento humano.

Las tendencias epidemiológicas nacionales, al igual que en el resto del mundo, han reforzado esta área del bienestar y la calidad de vida, lo cual por cierto involucra seriamente considerar el desarrollo planificado de profesionales especializados frente a estas realidades (Goldstein et al., 2011). No obstante, esto significa modificar los procesos de formación tradicional amparados en la racionalidad tecnológica, obsecuentes y reproductores, inermes a niveles formativos de capacitación que prescinden de la complejidad de los fundamentos epistemológicos involucrados. De sostener inmutables posiciones gremiales, seguirá el control biopolítico ajeno a los intereses de la población subsidiaria.

Los estudiantes de forma unánime valoraron los efectos del pensamiento propio, pero sería aventurado predecir la totalidad de los efectos, ya que a la base de una especialización no opera la totalidad de la evidencia acumulada. Por defecto, es el especialista en su trayectoria quien determinará los alcances que sea capaz de lograr con su formación. De este modo si bien los estudiantes reconocen estas dos últimas categorías con alta frecuencia (consecuencias del accionar y efectos del pensamiento propio), ambas están directamente derivadas de la provocación que resulta de las categorías previas (epistemología propia y fundamentos disciplinares) que imprimen la fuerza de la argumentación conceptual a las relaciones obtenidas.

No menos significativa resulta la asociación que se establece en función de la pertinencia de las herramientas, las cuales pueden ser más efectivas en la lógica de materializar la sostenibilidad de los argumentos de especialización. Por tanto, si se desea consolidar un ordenamiento conceptual para fines formativos de especialidad en fisioterapia/kinesiología, se sugiere no renunciar a considerar el carácter de las categorías obtenidas por el análisis de contenido cuantitativo y cualitativo del estudio (Figura 2). Máxime, cuando se trata de una actuación profesional con alcances formativos.

Finalmente, la categoría herramientas propias rescata el dogma de que hacerse preguntas sin tener sus propios instrumentos determina la dependencia del intelecto. Así de claro, la reflexión epistemológica en interacción con la praxis experta del especialista, construye realidades situadas que consolidan posturas, posturas muy alejadas de la neutralidad técnica. Posturas que pasan a 
transformarse en herramientas argumentales que devolverán una práctica autorregulada, participativa y resolutiva. Si así lo deciden los fisioterapeutas/kinesiólogos especialistas que incursionen en el uso de la innovación de herramientas, se debe alertar en los procesos de formación de especialistas que no consideren estratégica y tácticamente la reflexión de los aspectos epistemológicos, que indefectiblemente serán los derechos de la población por sobre las aspiraciones gremiales las cuales se encargarán de mantener la fidelidad de los profesionales a los intereses de situs y status social.

\section{Conclusión}

Frente a la solicitud de argumentar la necesidad de formación, los estudiantes del programa de especialidad en fisioterapia/kinesiología se pronuncian en un eje de racionalidad que establece una directa relación entre la consideración de los principios epistemológicos de la disciplina y la construcción de fundamentos, los cuales a su juicio son indispensables para develar las consecuencias, los efectos y las herramientas sustantivas para la actuación profesional.

Un aspecto que no fue posible garantizar en el presente estudio es la heterogeneidad de la muestra, dado que se trata de estudiantes participantes por conveniencia y que fueron sometidos a un proceso de estandarización en su selección, por lo que el resultado con alta probabilidad en su validez externa es limitado. Atendido el argumento, el estudio muestra que la incorporación de elementos reflexivos en la formación técnica no solo es atingente desde una concepción disciplinar, sino que es prioritario considerarlo por las consecuencias que exhibe para la responsabilidad social de las entidades que tienen la potestad de formar profesionales críticos para un país que desea garantizar los roles de la institucionalidad y la academia en un ámbito de convivencia democrática.

\section{Referencias}

Althusser, L. (1989). Ideología y aparatos ideológicos del estado (Notas para una investigación). En La filosofía como arma de la revolución (pp. 102-151). México D. F., México: Siglo XXI.

American Physical Therapy Association. (2015). Physical therapist practice and the movement system. Recuperado de https://www.apta.org/patient-care/interventions/movement-system-management/movement-systemwhite-paper

Artigas, A. (2012). Respiratory critical care HERMES syllabus: defining competencies for respiratory doctors. European Respiratory Journal, 39, 1294-1297. https://doi.org/10.1183/09031936.00056212 
Atria, F. (2014). Derechos sociales y educación: un nuevo paradigma de lo público. Santiago de Chile, Chile: Ediciones LOM.

Emol. (2017). Escasas oportunidades: Conoce las carreras más saturadas en el mercado laboral chileno. El Mercurio Online. Recuperado de: https://www.emol.com/noticias/Nacional/2017/12/29/889184/Conoce-las-carrerascon-mayores-indices-de-saturacion-en-el-mercado-laboral-chileno.html

Bourdieu, P. y Passeron J. C. (1995). La reproducción: Elementos para una teoría de la enseñanza. Barcelona, España: Laia S.A.

Canales, M. (2014). Metodologías de investigación social: introducción a los oficios. Santiago de Chile, Chile: Ediciones LOM.

Chiappa, P., y Muñoz-García, A. L. (2015). Equidad y capital humano avanzado: Análisis sobre las políticas de formación de doctorado en Chile. Psicoperspectivas, 14(3), 17-30.

CONAKI. (6-7-8 septiembre 2018). Compartiendo conocimiento para una salud de calidad. Presentado en el XXI Congreso Nacional de Kinesiología. La serena-Coquimbo, Chile.

De Ibarrola, M., Sañudo L., Moreno, M. G., y Barrera, M. E. (2012). Los profesionales de la educación con formación de posgrado que México requiere. México D. F., México: IPN/Universidad Autónoma de Yucatán/Red de Posgrado en Educación/REDMIIE/ANEFEP.

Delgado-Domínguez, J. M., y Gutiérrez, J. (1999). Métodos y técnicas cualitativas de investigación en ciencias sociales. Madrid, España: Editorial Síntesis.

Flick, U. (2007). Introducción a la Investigación Cualitativa. Madrid, España: Morata.

Gibbs, G. (2012). El análisis de datos cualitativos en investigación cualitativa. Madrid, España: Morata.

Goldstein, M. S., Scalzitti, D. A., Craik, R. L., Dunn, S. L., Irion, J. M., Irrgang, J., Kolobe, T... Shields, R. K. (2011). The revised research agenda for physical therapy. Physical Therapy, 91(2), 165-174. https://doi.org/10.2522/ptj.20100248

Gramigna, A. (2005). La epistemología de la diferencia en la formación educativa. Perfiles Educativos, 27(1), 70-94. Recuperado de http://www.scielo.org.mx/scielo.php?script=sci_arttext\&pid=S0185-26982005000100005

Jara, J. (2019). Bioética: ¿Una estrategia biopolítica? (Tesis de Maestría). Universidad de Valparaíso, Chile.

Ley 725. Código Sanitario. (1967). Ministerio de Salud, Chile. Recuperado de https://www.minsal.cl/wpcontent/uploads/2019/01/C.-Sanitario-Titulo-Preliminar-y-Libro-V.pdf

Maureira-Pareja, H. (2017). Síntesis de los principales elementos del Modelo Función - Disfunción del Movimiento Humano. REEM, 4(1), 7-24.

Medina-González, P., Muñoz-Cofré, R., Tapia-Gallardo, H., y Escobar-Cabello, M. (2014). Autonomía profesional del kinesiólogo: estrategias de problematización para el diagnóstico en contextos de un adulto mayor postrado. REEM, 1(1), 33-41.

Ministerio de Salud. (29 de enero de 2019). Proyecto: Modificación Código Sanitario, Libro V, "Del ejercicio de la medicina y profesiones afines". Consejo Asesor [Presentación PowerPoint]. Recuperado de https://www.minsal.cl/wp-content/uploads/2019/01/Presentaci\%C3\%B3n-Consejo-Asesor-29012019.pdf 
Muñoz-Cofré, R., Maureira-Pareja, H., Medina-González, P., Pinochet-Urzúa, R., Villarroel, G., Jalil, Y., SantanderCabello, R., y Escobar-Cabello, M. (2017). El movimiento del sistema ventilatorio en la función-disfunción humana: Fundamentos de especialidad. Talca, Chile: Universidad Católica del Maule.

Organización Panamericana de la Salud. (2019). Salud Universal en el siglo XXI: 40 años de Alma-Ata. Informe de la Comisión Alto Rivel. Recuperado de https://iris.paho.org/bitstream/handle/10665.2/50960/9789275320778_spa.pdf?sequence=5\&isAllowed=y

Pecarevic-Muñoz, M. (2017). DENAKE. Colegio de Kinesiólogos de Chile. Recuperado de: http://www.ckch.cl/colkine/denake/

Pitta, F., Mitchell, S., Chatwin, M., Clini, E., Emtner, M., Gosselink, R., Grant, K.M., Inal-Ince, D., Lewko, A., Oberwaldner, B., Williams, J., y Troosters, T. (2014). A core syllabus for post-graduate training in respiratory physiotherapy. Breathe, 10, $220-228$.

Rodríguez, B. J., Moreno, V. C., Plaza, M. M., y Retamal, Y. C. (2017). Kinesiterapia Respiratoria en Pediatría. Revista Pediatría Electrónica, 14(1), 26-34. Recuperado de: http://www.revistapediatria.cl/volumenes/2017/vol14num1/pdf/KINESOTERAPIA_RESPIRATORIA_PE DIATRIA.pdf

Román, O., Señoret, M. (2008). Estado actual de las especialidades médicas en Chile: realidad en el sistema público no municipalizado. Revista Médica de Chile, 136, 99-106.

Schön, D. (2010). La formación de profesionales reflexivos: Hacia un nuevo diseño de la enseñanza y el aprendizaje en las profesiones. Madrid, España: Paidós/MEC.

Strauss, A. y Corbin, J. (2012). Bases de la investigación cualitativa. Técnicas y procedimientos para desarrollar la teoría fundamentada. Medellín, Colombia: Universidad de Antioquía.

Vite-Pérez, M. (2000). La corrosión del carácter. Las consecuencias personales del trabajo en el nuevo capitalismo. Sociológica, 15(44), 229-232.

World Physiotherapy. (2020). Specialisation. Recuperado de https://world.physio/policy/ps-specialisation

Agradecimientos: A los kinesiólogos participantes del programa de especialidad en Función-Disfunción Ventilatoria 2018, por su contribución al desarrollo del ejercicio académico reflexivo.

\section{Contribución autoral}

a) Concepción y diseño del trabajo; b) Adquisición de datos; c) Análisis e interpretación de datos; d) Redacción del manuscrito; e) revisión crítica del manuscrito.

M. E. C. ha contribuido en a, b, c, d, e. H. M. P. ha contribuido en a, b, c, d, e.

Editora científica responsable: Mag. Florencia de León 